\title{
Reminiscence groupwork and autobiographical memory as part of meaningful activities
}

\author{
Andrew P. Allen ${ }^{1,2}$, Mary Lee Tully ${ }^{3}$, \\ Desmond O'Neill ${ }^{4}$, and Richard A.P. Roche ${ }^{1}$
}

\begin{abstract}
The current paper describes a reminiscence group activity session held as part of meaningful activities engagement for older adults. Topics of reminiscence included both autobiographical memories and memories of broader historical events from the past. Participants included those with memory impairment and those without, and participants with healthy memory were helpful in prompting memories in participants with memory impairment. Semantic and episodic autobiographical memory were assessed at baseline and following the end of both group activities, using the Episodic Autobiographical Memory Interview (EAMI) and quality of life was assessed using the Quality of Life AD-scale (QOL-AD). The reminiscence intervention did not significantly affect autobiographical memory recall or quality of life. However, oral reminiscence was reported to have increased outside of the reminiscence sessions.
\end{abstract}

Keywords: groupwork; group work; memory; older adults; reminiscence

1. Department of Psychology, Maynooth University, Republic of Ireland

2. Current affiliation: Trinity Centre for Ageing and Intellectual Disability, School of Nursing and Midwifery, Trinity College Dublin, Republic of Ireland

3. Peamount Healthcare, Newcastle, Co. Dublin, Republic of Ireland

4. Tallaght University Hospital and Trinity Centre for Health Sciences, Trinity College Dublin, Republic of Ireland

Address for correspondence: Richard A.P. Roche.richard.roche@mu.ie

Date of first (online) publication: 3rd December 2021

Acknowledgements: The work described in this manuscript was supported by a New Horizons grant from the Irish Research Council (REF: REPRO BU 2050158). The authors gratefully acknowledge the staff at the service provider who helped to facilitate the reminiscence groups. 


\section{Introduction}

There is existing evidence that structured reminiscence can benefit autobiographical memory in older adults with memory impairment (Lopes, Afonso \& Ribeiro, 2016; Meléndez et al., 2017) and that greater engagement with reminiscence work can enhance autobiographical memory in people with dementia (Woods et al., 2016). There are various mechanisms through which reminiscence may improve autobiographical memory; besides rehearsal, reminiscence may improve memory by enhancing mood/reducing depression (see review by Allen et al., 2018). However, reminiscence therapy may not benefit autobiographical memory performance in healthy older adults without memory impairment (Allen et al., 2020; de Medeiros et al., 2011). Previous work has also demonstrated that structured reminiscence may improve quality of life in older adults with dementia (O'Shea et al., 2014) and older adults with cognitive impairment (Siverová and Bužgová, 2018) staying in long-term residential care. The impact of structured reminiscence on autobiographical memory retrieval and quality of life in groups that include participants with cognitive impairment and those without is of interest. Furthermore, a recent scoping review has found a paucity of research on day centres and the associated benefits of the activities conducted at day centres (Orellana et al., 2020). The value of reminiscence intervention groupwork in this particular context was thus of interest.

Benson (2010) outlines factors to consider in planning group work, including identifying the demand for the group and considering alternatives to groupwork. With regard to demand for the group, the first and last authors were involved in a broader project examining reminiscence and autobiographical memory in older adults, and were motivated to examine reminiscence in older adult participants. The second and third authors were responsible for providing day service users with meaningful activities. The participants in this project attended day services on a voluntary basis, and were motivated to engage in meaningful activities, although this might not necessarily mean they were interested in pursuing a reminiscence course per se, and a few service users at the service provider did decline to participate in the groups. With regard to considering alternatives, reminiscence can be done on an individual, one-to-one level, and the first author has done 
so with a previous client with specific memory complaints. However, the group setting was familiar to the participants in the current study, conducting reminiscence in a group allowed the participants to draw on one another's memories, and it was a more time-effective means of conducting reminiscence. We also included an active control group to allow for comparison between reminiscence and a group intervention that did not focus on memories.

The current study examined a reminiscence group and a group that focused on equivalent topics, but with a focus on the present and future. We assessed autobiographical memory and quality of life at baseline and after the end of each group. With regard to autobiographical memory, we assessed both episodic memory (memories about a specific incident at a particular time and place, for example, a 21st birthday party) as well as semantic memory (facts about autobiographical memory, such as the address of one's workplace).

\section{Method}

This research received ethical approval from the SJH/AMNCH Research Ethics Committee (REC Reference: 2017/11/03). Participants either provided consent themselves or else consent was provdied by the participant in collaboration with a carer. Some individuals at the service provider declined to participate, and this decision was respected. Participation could be withdrawn at any time, and some participants did decline to take part in follow-up research assessments or specific aspects of the intervention.

\section{Design}

A crossover design was employed, whereby each participant was offered both the reminiscence group and the active control group, with participants randomised to be offered either the reminiscence condition first followed by the control, or else the two groups but in the opposite order. At baseline and after each of the groups, participants completed an individual research visit to assess quality of life and autobiographical memory. 


\section{Participants}

A sample size of fifteen was required to demonstrate an effect sized $\mathrm{f}=0.35$, at $\alpha=0.05$, assuming a power of 0.8 (Faul et al., 2007). Seventeen people attended a baseline research assessment. One participant died shortly after their first research visit, while another was unable to complete any of the assessments. These two participants were excluded from any further analysis. Of the remaining fifteen participants, twelve were female and three were male. Mean age was 83.5 ( $\mathrm{SD}=5.5$, range = 75-95). All participants were White Irish, and none reported regular alcohol consumption or cigarette smoking. Unfortunately, many participants were unable to complete both of the follow-up research assessments due to health problems. Six participants completed all three assessments.

\section{Measures}

The Episodic Autobiographical Memory Interview (EAMI; Irish et al., 2008) was used to assess semantic and episodic autobiographical memory across the lifespan (the life epochs assessed are 0-15 years of age, 15-30 years, 31-45 years, 46 years of age - last 5 years, and the last 5 years), and a total score is calculated across the five epochs. We assessed quality of life with the Quality of Life-AD scale (QOL-AD; Logsdon et al. 1999; 2002). This is a 13-item scale where various aspects of quality of life are assessed via self-report, on a four-point Likert scale ("poor", "fair", "good", and "excellent").

\section{Reminiscence groups and setting}

The reminiscence and active control interventions were delivered in small groups at the service provider. This service provider (located in Ireland) places an emphasis on providing high-quality services to meet healthcare demands, with residents and clients from the local communities as active partners in their health and wellness. The service provider provides a secure and comfortable environment that offers meaningful, life-enhancing activities, so people of all abilities can better manage their health and wellbeing, learn new skills and develop friendships. Meaningful engagement includes a broad range of activities 
including exercise, musical programs, and gardening. There is a strong focus on rights and sharing of information from the multidisciplinary team, through regular workshops covering many topics, supporting people to be more included in their local community.

The reminiscence groups were facilitated by the first author along with members of support staff at the service provider. Groups were typically of six-eight people, but would break into sub-groups of 2-3 to allow for individuals to contribute more to the discussion. The groups comprised six weekly sessions, each lasting one hour. The reminiscence groups used a simple reminiscence approach, adapted from the "remembering yesterday, caring today" program (Schweitzer $\&$ Bruce, 2008). This program was used in previous research examining autobiographical memory as an outcome (Woods et al., 2016), and we had used a similar adaptation previously (Allen et al., 2020).

For the reminiscence groups, in addition to autobiographical memories, participants were prompted to discuss memories of historical events in the public sphere (such as John F. Kennedy's visit to Ireland in the 1960s). For the active control groups, the topics of discussion were approximately matched to the content of the reminiscence groups, but with a focus on the present and future. For example, the active control group discussed learning new things whilst the reminiscence group discussed schooldays. The weekly topics for the reminiscence group and active control group are outlined in Table 1 below.

Table 1

Session topics for reminiscence group and active control

\begin{tabular}{|l|l|l|}
\hline & Reminiscence sessions & Current life sessions \\
\hline Session 1 & $\begin{array}{l}\text { Introduction, childhood and } \\
\text { family life }\end{array}$ & $\begin{array}{l}\text { Introduction, health and well- } \\
\text { being }\end{array}$ \\
\hline Session 2 & Schooldays & $\begin{array}{l}\text { Continued education/learning } \\
\text { new skills }\end{array}$ \\
\hline Session 3 & Historical session A & Current affairs (national) \\
\hline Session 4 & $\begin{array}{l}\text { Homes, gardens and animals, } \\
\text { going out and having fun }\end{array}$ & Holidays and celebrations \\
\hline Session 5 & Historical session B & Current affairs (international) \\
\hline Session 6 & $\begin{array}{l}\text { Weddings, babies, children, } \\
\text { rounding up }\end{array}$ & $\begin{array}{l}\text { Family life, grandchildren, } \\
\text { relationships, rounding up }\end{array}$ \\
\hline
\end{tabular}


Participants were encouraged to listen to recordings in an online archive at the Digital Repository of Ireland (available at https://repository.dri.ie/catalog/wp98p078n). This archive was a collection of verbal reminiscences provided by healthy older adults in a previous project (see Allen et al., 2020). It was determined during the ethics process not to record the reminiscence sessions in the current project, as participants with memory problems may not have capacity to give fully informed consent to having their memories shared in an online archive. Once all the group sessions and research visits had finished, there was a presentation ceremony where all participants received a certificate to acknowledge their contribution to the project.

\section{Results}

Although episodic autobiographical memory was slightly higher following the reminiscence group, there was no significant effect of reminiscence intervention for those who completed all assessments, either for episodic memory, $F(2,10)=0.75, p=.5$, partial eta squared $=0.13$, or semantic memory, $F(2,10)=1.2, p=.34$, partial eta squared $=0.2$ (see Figure 1).

Figure 1: EAMI score at baseline, post-active control and post-reminiscence for (A). episodic memory and (B). semantic memory. Error bars represent standard error of the mean.

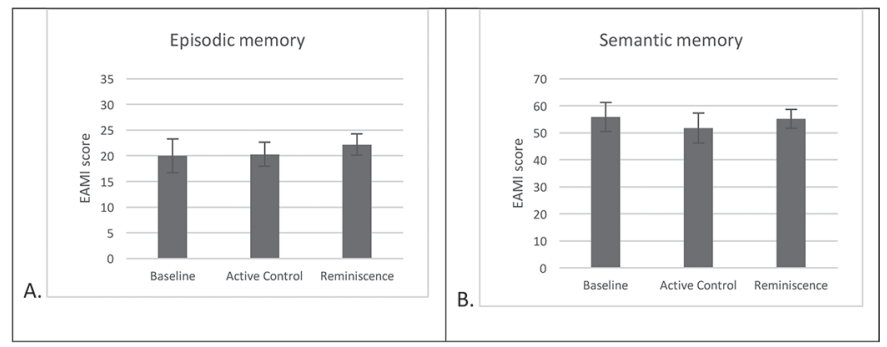

There was no significant difference in quality of life following reminiscence therapy in those who completed both groups, $F(2,8)=$ $0.18, p=.84$, partial eta squared $=0.04$ (see Figure 2). 
Figure 2: QoL-AD score at baseline, post-active control and postreminiscence. Error bars represent standard error of the mean.

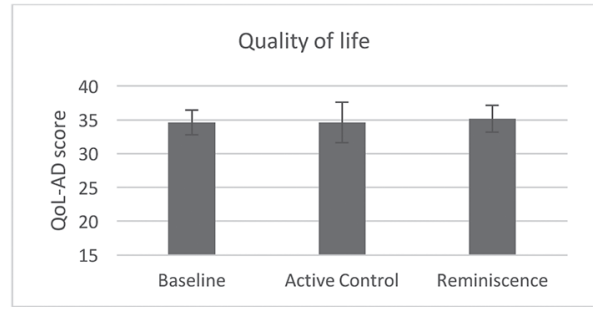

\section{Discussion}

The current project did not find a significant effect of reminiscence on autobiographical memory or quality of life compared to an active control. There was a high level of dropout from the study, so the final sample was lacking in statistical power to detect an effect of the intervention, and given the limited sample size there was a lack of statistical power to detect any effect of the order in which participants completed the interventions. The participants who completed all assessments tended to have better baseline level of performance, and, as noted above, people with good autobiographical memory do not show a clear benefit of structured reminiscence on autobiographical memory retrieval (Allen et al., 2020; de Medeiros et al., 2011). Nonetheless, although semantic memory was quite high in the participants at baseline, it was not so strong for episodic memory, suggesting that the lack of an effect of group participation is not simply due to autobiographical memory already being at ceiling level.

Notwithstanding this lack of an effect at assessment, it was noted anecdotally by staff that participants discussed a wider range of autobiographical memories following the reminiscence groups. For example, when some of the participants went home to their long stay accommodation or to their home in the community with their families, they continued to reminisce, and in some cases recalled more information as time went on. It appears that the activity opened up more time for engaging with autobiographical memory on an ongoing 
basis. Families and staff who linked in with people from the group explained how it seemed to help them cope with growing older, as they recreated the meaning of their lives through the stories they shared and, potentially, via the stories they did not share in the group, but later divulged at home, where they felt more confident to do so. Despite the lack of a significant effect on reported quality of life post-intervention, many participants reported a sense of achievement about their lives when reflecting on their autobiography, and a "feel-good factor" prevailed for a while later into the evening following group sessions. Some continued their conservations by telephone afterwards, and the sharing of stories continued beyond the scheduled group session. It is thus likely that there were some autobiographical memory effects that were not detected by the post-reminiscence research assessment. To this end, future research may be more likely to detect such effects using informant report about participant's quality of life and memory before and after intervention. It is also possible that a longer/more extensive reminiscence intervention may have had more visible effects in a larger cohort, and where possible the reminiscence intervention can draw on resources from family members, carers and the community to further personalise the reminiscence process.

Although participants generally reported enjoying the reminiscence activities, some reported finding the research assessments aversive. These research assessments (including the autobiographical memory interview) were done one-on-one, where specific questions were posed of the individual. Although one-one-one assessments are empirically useful in assessing individuals, in that they avoid the risk of being prompted by peers, the fact that the assessments leave far less scope for a person with memory problems to "gloss over" gaps in autobiographical memory compared to the reminiscence group activity render them a less pleasant experience for participants. Nonetheless, one-on-one assessment also avoids any mistakes being visible to one's peers when completing a demanding assessment, and is thus likely to be less aversive than an assessment in a group setting would be. This should be borne in mind if combining reminiscence groups with a research aspect that includes assessment of memory.

Although the first author was previously unknown to the participants, the support workers at the service provider were more familiar with the participants, and helped ensure they were at ease during the 
groups. Some of the participants themselves had had a more longterm friendships with each other, and were sometimes able to prompt autobiographical memories in their peers at a more specified level than anyone else. However, facilitating the groups does require staff time, and sufficient staff time should be taken into account when planning reminiscence activities.

There was generally a lack of disagreement between participants during the group sessions, and where this did happen it was transient, and tended to happen during discussion of current affairs sessions in the sessions focused on current life, rather than during the reminiscence sessions. However, even though the current work emphasised positive memories, it should be borne in mind that discussions of the past as well as the future and present can lead to arguments between group members. Benson (2010) has noted that conflict or disagreement between group members can be used as material to work with in a group setting, and may even be of therapeutic benefit if certain issues can be worked through. However, disagreements should not dominate a discussion where the aim is to generate autobiographical memories.

A recent scoping review (Orellana et al., 2020) highlights evidence gaps on perceptions of day centres and what they can offer to wider stakeholders, although some work has been done on social and preventative services. They cite work that emphasises the value of integrating health and social services for older adults, while at the same time acknowledging that such integration is challenging, given factors such as differences in organisational culture, lack of resources and difficulties in managing program expansion (Vogel et al., 2007). Future group-based research should seek to address these gaps, not just for reminiscence, but for other group activities that service providers organise.

The current research was conducted prior to the outbreak of the COVID-19 pandemic. Since then, the service provider has continued to engage with service users onsite where possible, but has had to alter its logistics to ensure that infection risk is minimised. Service users who are vulnerable to infection may be supported in using virtual platforms to engage with staff; where possible, this can be a means of incorporating multimedia historical materials to prompt memory (e.g. sharing screens to display photographs from earlier periods of life). The service provider provides reminiscence as one part of a selection of day services, including assistive technology with mobility and 
physiotherapy, therapeutic gardening, diet and exercise, preventing frailty, and occupational therapy support.

In conclusion, reminiscence groupwork can be an enjoyable meaningful activity for older adults, though it did not seem to significantly enhance autobiographical memory performance or quality of life in this sample. Structured reminiscence groupwork may also lead to a greater level of habitual reminiscence in day service users; this would be an interesting research question for further observational study.

\section{References}

Allen, A.P., Doyle, C., Commins, S. and Roche, R.A.P. (2018) Autobiographical memory, the ageing brain and mechanisms of psychological interventions. Ageing Research Reviews, 42, 100-111

Allen, A.P., Doyle, C. and Roche, R.A.P. (2020) The impact of reminiscence on autobiographical memory, cognition and psychological well-being in healthy older adults. Europe's Journal of Psychology, 16, 2, 317-330

Benson, J. (2010) Working more creatively with groups (3rd ed.). London: Routledge De Medeiros, K., Mosby, A., Hanley, K.B., Pedraza, M.S. and Brandt, J. (2011) A randomized clinical trial of a writing workshop intervention to improve autobiographical memory and well-being in older adults. International Journal of Geriatric Psychiatry, 26, 8, 803-811

Faul, F., Erdfelder, E., Lang, A.G. and Buchner, A. (2007) G* Power 3: A flexible statistical power analysis program for the social, behavioral, and biomedical sciences. Behavior Research Methods, 39, 2, 175-191

Irish, M., Lawlor, B.A., O'Mara, S.M. and Coen, R.F. (2008) Assessment of behavioural markers of autonoetic consciousness during episodic autobiographical memory retrieval: A preliminary analysis. Behavioural Neurology, 19, 1-2, 3-6

Logsdon, R.G., Gibbons, L.E., McCurry, S.M. and Teri, L. (1999) Quality of life in Alzheimer's disease: patient and caregiver reports. Journal of Mental Health and Aging, 5, 21-32

Logsdon, R.G., Gibbons, L.E., McCurry, S.M. and Teri, L. (2002) Assessing quality of life in older adults with cognitive impairment. Psychosomatic Medicine, 64, 3, 510-519

Lopes, T.S., Afonso, R.M.L.B.M. and Ribeiro, O.M. (2016) A quasi-experimental 
study of a reminiscence program focused on autobiographical memory in institutionalized older adults with cognitive impairment. Archives of Gerontology and Geriatrics, 66, 183-192

Melendez, J.C., Torres, M., Redondo, R., Mayordomo, T. and Sales, A. (2017) Effectiveness of follow-up reminiscence therapy on autobiographical memory in pathological ageing. International Journal of Psychology, 52, 4, 283-290

Orellana, K., Manthorpe, J. and Tinker, A. (2020) Day centres for older people: a systematically conducted scoping review of literature about their benefits, purposes and how they are perceived. Ageing \& Society, 40, 1, 73-104

O'Shea, E., Devane, D., Cooney, A., Casey, D., Jordan, F., Hunter, A., Murphy, E., Newell, J., Connolly, S. and Murphy, K. (2014) The impact of reminiscence on the quality of life of residents with dementia in long-stay care. International Journal of Geriatric Psychiatry, 29, 10, 1062-1070

Schweitzer, P. and Bruce, E. (2008) Remembering yesterday, caring today: Reminiscence in dementia care: A guide to good practice. London: Jessica Kingsley Siverová, J. and Bužgová, R. (2018) The effect of reminiscence therapy on quality of life, attitudes to ageing, and depressive symptoms in institutionalized elderly adults with cognitive impairment: A quasi-experimental study. International Journal of Mental Health Nursing, 27, 5, 1430-1439

Woods, R.T., Orrell, M., Bruce, E., Edwards, R.T., Hoare, Z., Hounsome, B., Keady, J., Moniz-Cook, E., Orgeta, V., Rees, J. and Russell, I. (2016) REMCARE: pragmatic multi-centre randomised trial of reminiscence groups for people with dementia and their family carers: effectiveness and economic analysis. PloS one, 11, 4, p.e0152843. 